\title{
Study of natural aging on nanoparticles and their strengthening effect in Al-Mg-Si alloy
}

\section{Zhenjie Cui}

Institute of Metal Research Chinese Academy of Sciences

Haichang Jiang ( $\nabla$ hcjiang@imr.ac.cn )

Institute of Metal Research, Chinese Academy of Science

Duo Zhang

Institute of Metal Research Chinese Academy of Sciences

Yuanyuan Song

Institute of Metal Research Chinese Academy of Sciences

Desheng Yan

Institute of Metal Research Chinese Academy of Sciences

Lijian Rong

Institute of Metal Research Chinese Academy of Sciences

\section{Nano Express}

Keywords: Al-Mg-Si alloy, natural aging, nanoparticles

Posted Date: August 19th, 2021

DOI: https://doi.org/10.21203/rs.3.rs-806071/v1

License: (c) (i) This work is licensed under a Creative Commons Attribution 4.0 International License.

Read Full License 
1 Study of natural aging on nanoparticles and their strengthening effect in Al-Mg-Si

2 alloy

3 Zhenjie Cui ${ }^{1,2,3}$, Haichang Jiang ${ }^{1,3 *}$, Duo Zhang ${ }^{1,2,3}$, Yuanyuan Song ${ }^{1,3}$, Desheng Yan

$4 \quad 1,3$, Lijian Rong ${ }^{1,3 *}$

6 Abstract

Atom probe tomography (APT) and transmission electron microscopy (TEM) were employed simultaneously to study nanoparticles and their strengthening effect in artificial

9 aging (AA) Al-Mg-Si alloy with different natural aging (NA). We discovered that NA leads to formation of different type of nanoparticles meanwhile affect their fraction and

11 dimension. Separation of the contribution from different types of nanoparticles to the 12 alloy strength is conducted. In LNA-AA, $\beta^{\prime}$ with a volume fraction of $0.16 \%$ contributions

$1354 \mathrm{MPa}$ to the yield strength while $0.14 \% \beta^{\prime \prime}$ contributes $101 \mathrm{MPa}$, which fully 14 demonstrates that $\beta^{\prime \prime}$ is the main strengthening phase. The thermodynamic analysis was 15 conducted for further research and the differential scanning calorimeter (DSC) results 16 show that LNA forms more numerous clusters I than SNA and consumes more vacancies 17 and solute atoms then does not have enough driving force to precipitate clusters II which are the nucleation sites of nanoparticles. When there is a certain distance between the

19 nucleation sites, preferential growth of few $\beta^{\prime \prime}$ occur then converted to $\beta^{\prime}$. The solute

20 atoms in the region nearly which there is no nucleation site do not have enough driving 
force to diffuse and precipitate but stay in the matrix, reducing the volume fraction of the nanoparticles.

Keywords: Al-Mg-Si alloy; natural aging; nanoparticles

\section{Background}

The precipitation sequence of the nanoparticles in the heat-treatable reinforced Al$\mathrm{Mg}$-Si alloys is generally considered as a supersaturated solid solution (SSSS) $\rightarrow$ Solute clusters $\rightarrow$ GP zones $\rightarrow \beta^{\prime \prime} \rightarrow \beta^{\prime} /$ Type A/Type B/Type $C \rightarrow \beta[1-4]$. Previous studies have found that natural aging (NA) strongly affects the nanoparticles after artificial aging (AA) and negatively affects the alloy's properties. Røyset, Jostein et al. have revealed that NA 3600 hours leads to a lowering in the number density of AA nanoparticles and a decrease in the yield strength by $30 \mathrm{MPa}$ in an Al-Mg-Si alloy [5]. Tao, G. H. et al. proposed that NA 30 days reduces the AA peak hardness by $13 \mathrm{HV}$ in an $\mathrm{Al}-1.0 \mathrm{wt} \% \mathrm{Mg}-0.5 \mathrm{wt} \% \mathrm{Si}$ alloy which is related to the broad precipitate length distribution and appearance of large-sized $\beta^{\prime \prime}[6]$. Wenbin $\mathrm{Tu}$ et al. reported that the AA hardening rate was dramatically slowed down by NA 14 days because NA clusters delayed the formation of $\beta^{\prime \prime}$, leading to a drop of the peak hardness by $30 \mathrm{HV}$ in an Al-0.5at.\% Mg-1.0at.\% Si alloy [7]. Recently, we discovered that the NA also leads to the appearance of different types of nanoparticles so that the fractional and dimensional variation of each kind of nanoparticle can further affect the magnitude of precipitation strengthening. Transmission electron microscopy 
(TEM) is effective in determining the structure of nanoparticles [8, 9]. However, the distinguishing of different nanoparticles is difficult only by TEM due to the size limitation of clusters and GP zones as well as the similar needle-like morphology of $\beta^{\prime \prime}$ and $\beta^{\prime}$. The three-dimensional atom probe (3DAP) provides a near-atomic resolution and a capacity to record statistically meaningful data on the compositions, size, and volume fraction of nanoparticles $[10,11]$. In this paper, TEM and 3DAP were employed simultaneously to reveal the fractional and dimensional variation of different nanoparticles in an artificial aging Al-Mg-Si alloy with different NA. We calculated the strengthening effect of the contribution from different AA nanoparticles and consequently reveal the essence of decreased strength.

\section{Methods}

\section{Material}

An Al-Mg-Si alloy with a nominal composition of Al-0.95wt $\% \mathrm{Mg}-0.8 \mathrm{wt} \% \mathrm{Si}$ was prepared to study. The cast ingots were homogenized at $550^{\circ} \mathrm{C} / 10 \mathrm{~h}$ and forged into a bar with a diameter of $40 \mathrm{~mm}$. The aging treatments started with a solid solution treatment of $4 \mathrm{~h}$ at $525^{\circ} \mathrm{C}$, water-quenched to room temperature. After the solution, samples were stored at room temperature for a different time before experiencing a $175^{\circ} \mathrm{C} / 8 \mathrm{~h} \mathrm{AA}$.

\section{Characterization}

The tensile tests were performed on Shimadzu Autograph Dcs-10T Electronic universal testing machines. The TEM sample was prepared by a Struers Tenupol-5 type 
twin-jet electropolishing with a $30 \%$ methanol solution of nitric acid, and the microstructure was observed using a JEM 2100F transmission electron microscope. The APT samples of NA were prepared by electrolytic polishing and were completed within 10-15min. NA will not occur again in the APT sample after the preparation is completed which was proved by [12]. The APT experiments were carried out on LEAP 5000 XR with laser mode at a temperature of $25 \mathrm{~K}$ and a laser pulse frequency of $200 \mathrm{kHz}$. The 3D reconstruction and analysis are performed using IVAS 3.8.2 software. The thermal analysis was carried out on Q1000 DSC and the heating rate is $10^{\circ} \mathrm{C} / \mathrm{min}$.

\section{Results and discussion}

The NA hardness curve of the Al-Mg-Si alloy is shown in Fig.1 (a), which is drawn by averaging seven hardness indentation data. The NA hardness firstly increases fast and then tends to be stable as the NA time reaches $24 \mathrm{~h}$. Samples of NA10min, $2 \mathrm{~h}, 24 \mathrm{~h}$ indicated by the black arrows on the NA hardness curve are selected for investigation, named SNA, MNA, and LNA, respectively. After experiencing a $175^{\circ} \mathrm{C} / 8 \mathrm{~h} \mathrm{AA}$, the engineering stress-strain curves of SNA-AA, MNA-AA, and LNA-AA are presented in Fig.1(b). The yield strengths of SNA-AA, MNA-AA, and LNA-AA are $280 \mathrm{MPa}$, $253 \mathrm{MPa}$, and $230 \mathrm{MPa}$, respectively. According to the variation of strength, LNA-AA loses about $18 \%$ strength compared to the SNA-AA. It can be seen that NA makes the alloy strength obviously decrease. 

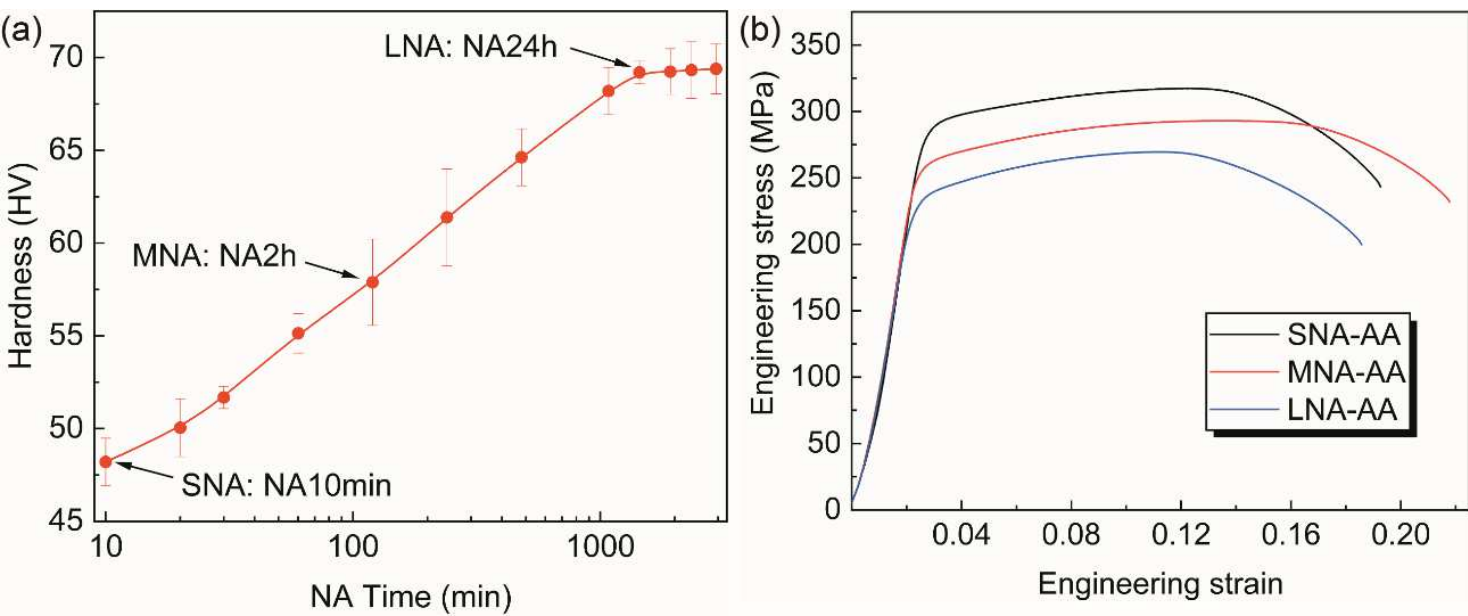

Fig.1 (a) NA hardness curves of the Al-Mg-Si alloy. (b) Engineering stress-strain curves of SNA-AA, MNA-AA, and LNA-AA.

The TEM bright-field images of SNA-AA, MNA-AA, and LNA-AA with the electron beam parallel along the $<001>_{\mathrm{Al}}$ are shown in Fig.2 (a) (c). The HRTEM images and FFTs viewed along with the $<001>_{\mathrm{Al}}$ are shown in Fig.2 (d) (f). The SNA-AA has a fine and uniform distribution of the nanoparticles as shown in Fig.2 (a). The nanoparticles become coarser in MNA-AA and nanoparticles over 100nm appeared. The length of nanoparticles of LNA-AA becomes longer and a few considerably elongated nanoparticles exceed $200 \mathrm{~nm}$ and the number of nanoparticles is significantly reduced. The FFT in Fig.2 (d) agrees well with the monoclinic $\beta "$ " [8]. Fig.2 (e), (f) show the FFTs of the considerably elongated nanoparticles which is consistent with hexagonal $\beta^{\prime}$ [9]. $\beta^{\prime \prime}$ and $\beta^{\prime}$ coexist in MNA-AA and LNA-AA. The TEM images realize the statistics of average radius $(R)$ of $\beta^{\prime \prime}$ and $\beta^{\prime}$ and the detailed data are shown in Table 1 . The $R$ of $\beta^{\prime \prime}$ is $1.93 \mathrm{~nm}$ in SNA-AA and $2.15 \mathrm{~nm}$ in LNA-AA, while for $\beta^{\prime}$ is $2.39 \mathrm{~nm}$ in MNA-AA and 

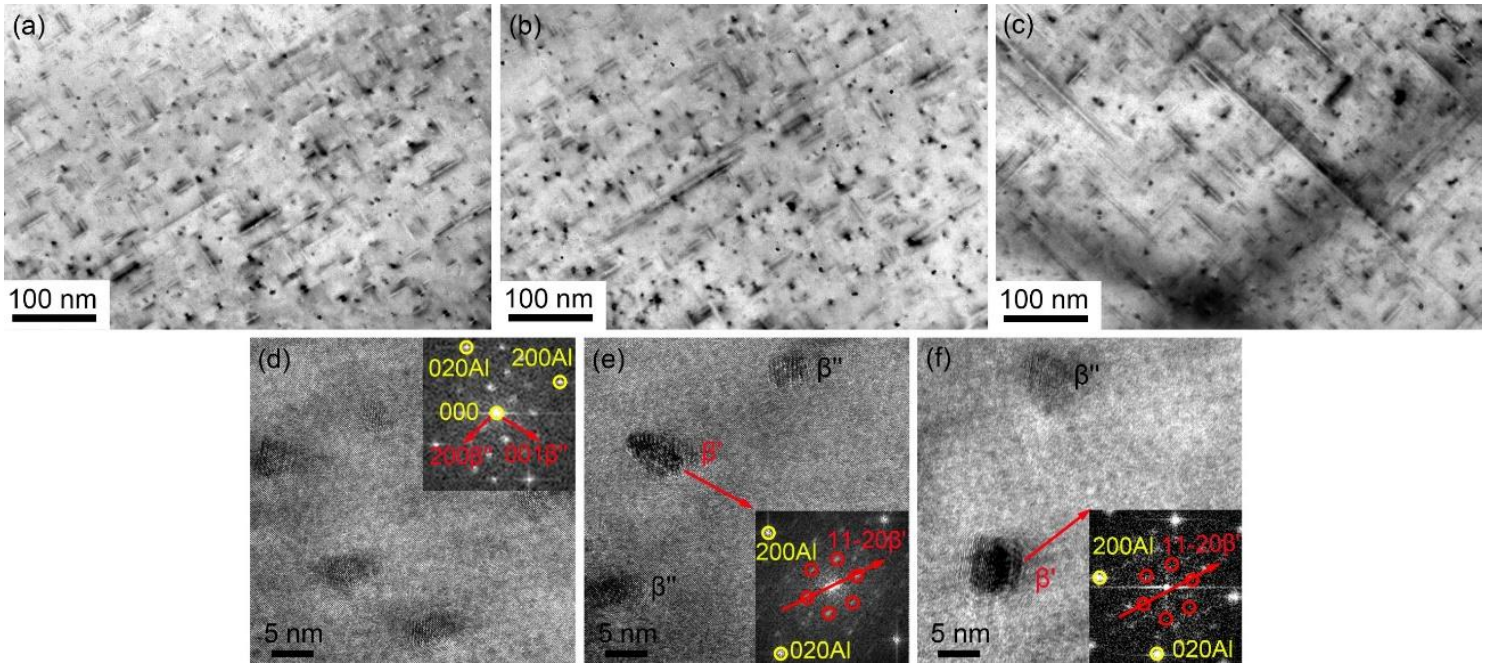

Fig.2 TEM bright-field images of (a) SNA-AA, (b) MNA-AA and (c) LNA-AA. HRTEM

images and FFTs of the $\beta^{\prime \prime}$ and $\beta^{\prime}$ of (d) SNA-AA, (e) MNA-AA, and (f) LNA-AA, and the images were taken with the electron beam along the $<001>_{\mathrm{Al}}$ orientation.

Fig.3(a), (b) show the $3 \mathrm{NN}$ distribution of the experimental and randomized data of

$\mathrm{Mg}$ and $\mathrm{Si}$ in SNA and $\mathrm{Mg}+\mathrm{Si}$ in LNA. Comparing (a) and (b), Mg and Si solute atoms

hardly aggregated in SNA while aggregated significantly in LNA. Fig.3 (c) and (d) show the 3D atom maps of evenly distributed $\mathrm{Mg}$ (red), $\mathrm{Si}$ (purple) of SNA, and $\mathrm{Mg}+\mathrm{Si}$ clusters of LNA. Obviously, the formation of NA clusters in LNA is an important reason for the degradation of AA strength. It is commonly believed that the formation of NA clusters depletes the super-saturated solutes in the matrix as well as not being favorable nucleation

110 sites for further nanoparticles [13, 14]. Instead, clusters that formed at early AA were confirmed to play the role of critical nuclei for $\beta^{\prime \prime}[6]$. 


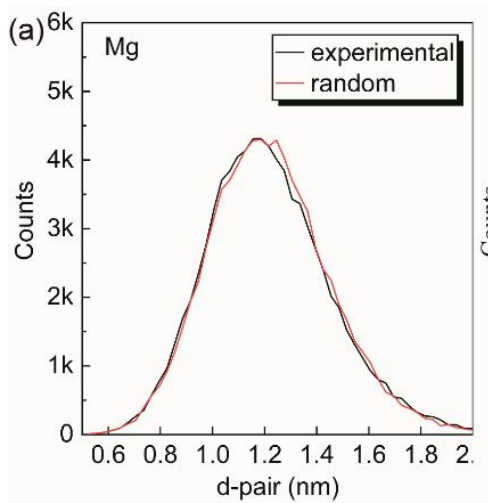

(c)
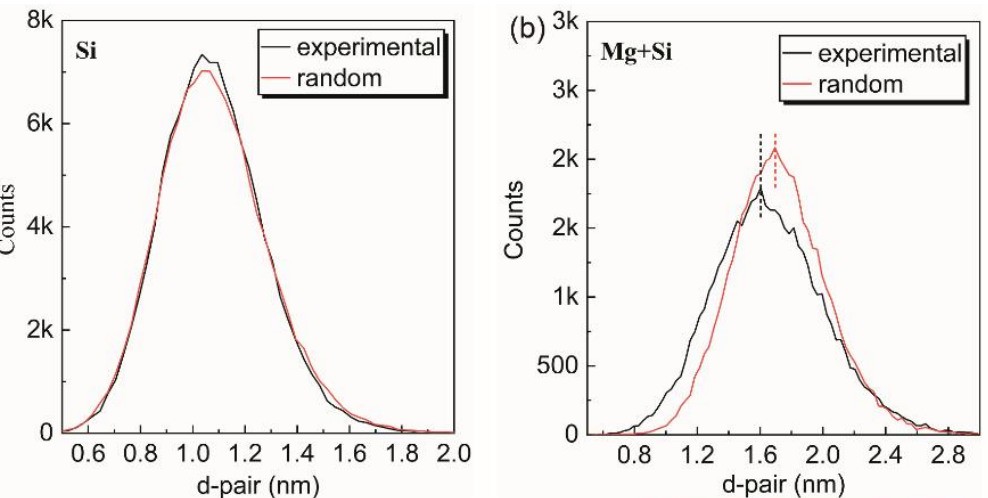

(d)

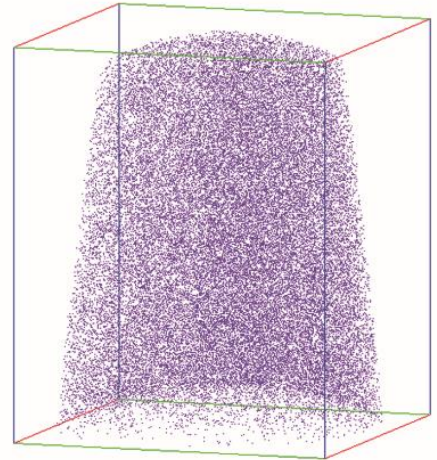

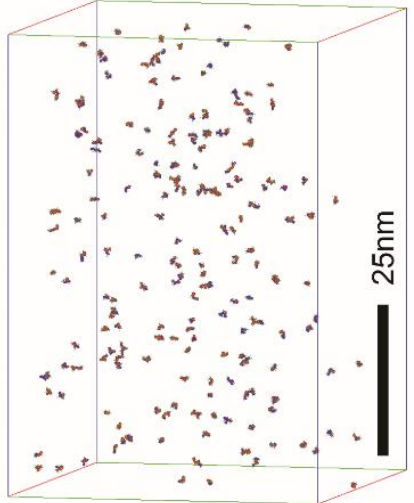

Fig.3 3D atom maps of $\mathrm{Mg}$ (red) and $\mathrm{Si}$ (purple) and the 3NN distribution of experimental and randomized data in the Al-Mg-Si alloy: (a), (c) SNA, (b), (d) LNA.

The 3D atom maps of $\mathrm{Mg}($ Orange), $\mathrm{Si}$ (purple), and a 10.0 at $\% \mathrm{Mg}+\mathrm{Si}$ isoconcentration surface of SNA-AA, MNA-AA, and LNA-AA are shown in Fig.4. The nanoparticles in the MNA-AA and LNA-AA are coarsened and reduced in number compared with SNA-AA which is consistent with the TEM results in Fig.2. The interparticle spacing in SNA-AA is small, that is, the nucleation sites of $\beta^{\prime \prime}$ are close to each other. The inter-particle spacing in MNA-AA becomes bigger and nanoparticles in LNAAA are sparsely distributed. As reported in Ref. [6], long NA leads to the preferential growth and the formation of large-sized $\beta "$ which created a large inter-particle spacing. 

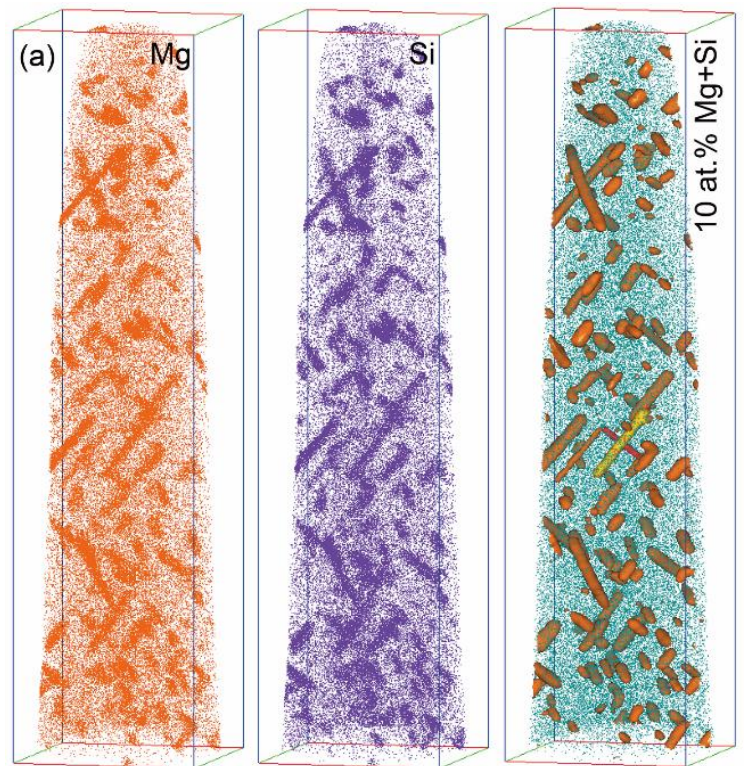

(c)

(b)
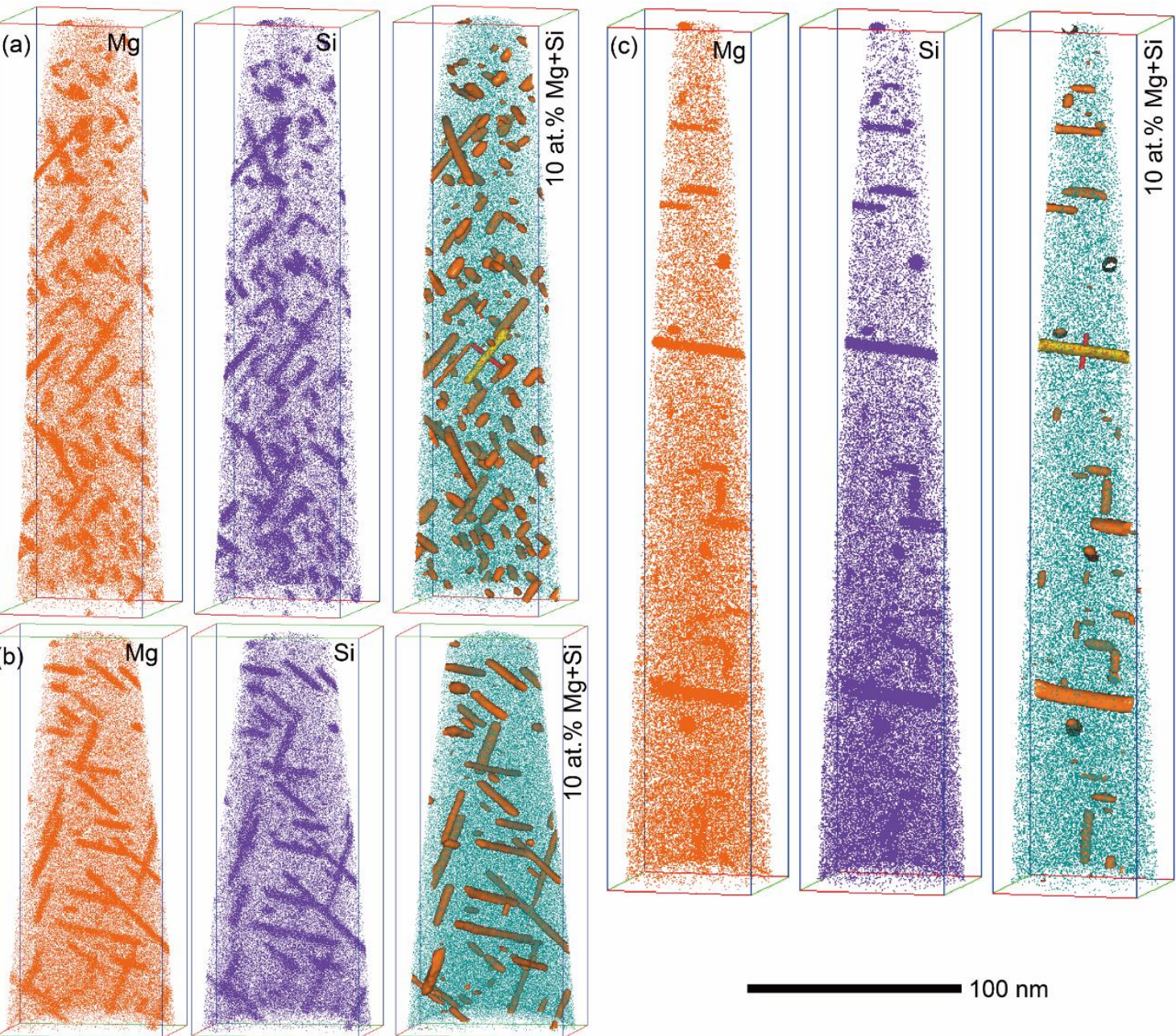

Fig.4 The 3D atom maps of $\mathrm{Mg}$, $\mathrm{Si}$ and a $10.0 \mathrm{at} \% \mathrm{Mg}+\mathrm{Si}$ iso-concentration surface of

(a) SNA-AA, (b) MNA-AA (c) LNA-AA, Al(Green), Mg(Orange), Si(purple). The

nanoparticles marked in yellow in (a), (c) were carried out 1D concentration analysis in the following part.

The maximum separation method is applied to distinguish the different nanoparticles of SNA-AA, MNA-AA, and LNA-AA according to previous studies with $\mathrm{d}_{\max }=0.7 \mathrm{~nm}$ and $\mathrm{N}_{\min }=10[10,15]$. The statistical data of different nanoparticles have been listed in Table 1. The Clusters and GP zones at AA state are collectively referred to as spherical nanoparticles, abbreviated as GC. It is considered that the supersaturation of solute and 
133 presence of vacancies is necessary for nucleation of GC during AA in small local regions 134 of the Al lattice between the large nanoparticles [16]. The volume fraction of GC is 135 increasing with the extending of NA time. A low fraction of GC in SNA-AA can be 136 attributed to greater consumption of the solute by densely distributed $\beta^{\prime \prime}$ with a volume 137 fraction of $0.74 \%$, thus reduced solute supersaturation of the matrix between the nanoparticles. The falling of the volume fraction of $\beta^{\prime \prime}$ from $0.74 \%$ in SNA-AA to $0.21 \%$ 139 in MNA-AA is dramatic and $0.22 \% \beta^{\prime}$ appear in MNA-AA. Obviously, $\beta^{\prime}$ precipitates at 140 the expense of $\beta^{\prime \prime}$. The $\beta^{\prime \prime}$ and $\beta^{\prime}$ become coarser in LNA-AA and the overall volume 141 fraction drop to $0.37 \%$ including $0.14 \% \beta^{\prime \prime}$ and $0.16 \% \beta^{\prime}$. Additionally, it can be obtained 142 by the 'Solute in matrix' that more solute atoms remain in the matrix at samples with 143 longer NA. This is probably because those numerous NA clusters deplete the super144 saturated solutes leading to the driving force of precipitation of solute atoms being 145 reduced so that more of them remain in the matrix.

146 Table 1. The relative data of nanoparticles in the different states

\begin{tabular}{ccccc}
\hline \multirow{2}{*}{ Samples } & Nanoparticles & $R / \mathrm{nm}$ & $V_{f}^{\mathrm{a}}$ & Solute in \\
\hline \multirow{2}{*}{ SNA-AA } & GC & $0.82 \pm 0.13^{\mathrm{a}}$ & $0.02 \%$ & $0.45 \mathrm{wt} \% \mathrm{Mg}$ \\
& $\beta^{\prime \prime}$ & $1.93 \pm 0.16^{\mathrm{b}}$ & $0.74 \%$ & \multirow{2}{*}{$0.26 \mathrm{wt} \% \mathrm{Si}$} \\
\cline { 2 - 5 } MNA-AA & Sum & - & $0.76 \%$ & \\
& GC & $0.77 \pm 0.12^{\mathrm{a}}$ & $0.05 \%$ & \multirow{2}{*}{$0.52 \mathrm{wt} \% \mathrm{Mg}$} \\
& $\beta^{\prime \prime}$ & $1.95 \pm 0.06^{\mathrm{b}}$ & $0.21 \%$ & \multirow{2}{*}{$0.31 \mathrm{wt} \% \mathrm{Si}$} \\
& $\beta^{\prime}$ & $2.39 \pm 0.1^{\mathrm{b}}$ & $0.22 \%$ & \\
\cline { 2 - 5 } & Sum & - & $0.48 \%$ & \\
\hline
\end{tabular}




\begin{tabular}{lcccc} 
& $\beta^{\prime \prime}$ & $2.15 \pm 0.11^{\mathrm{b}}$ & $0.14 \%$ & $0.61 \mathrm{wt} \% \mathrm{Mg}$ \\
LNA-AA & $\beta^{\prime}$ & $3.23 \pm 0.27^{\mathrm{b}}$ & $0.16 \%$ & \\
& Sum & - & $0.37 \%$ & $0.35 \mathrm{wt} \% \mathrm{Si}$ \\
\hline
\end{tabular}

$147 \quad{ }^{a}$ Parameters determined from the APT data.

$148{ }^{\mathrm{b}}$ Average radius $R$ of $\beta^{\prime \prime}$ and $\beta^{\prime}$ were measured in the bright-field images in Fig. 2.

Fig.5 (a), (b) demonstrate the 1D concentration profiles of representative $\beta^{\prime \prime}$ and $\beta^{\prime}$

marked in yellow in Fig.4 (a) and (c), respectively. The 1D concentration profiles which were measured by a selected cylinder with a moving step of $0.3 \mathrm{~nm}$ distinguished $\beta^{\prime \prime}$ and content of $\mathrm{Al}$ is 32.13 at.\% and 19.22 at.\%, respectively. It indicates that the transformation process from $\beta^{\prime \prime}$ to $\beta^{\prime}$ is dominated by $\mathrm{Mg}$, and $\mathrm{Al}$ is continuously discharged which is consistent with Refs [10].

(a)

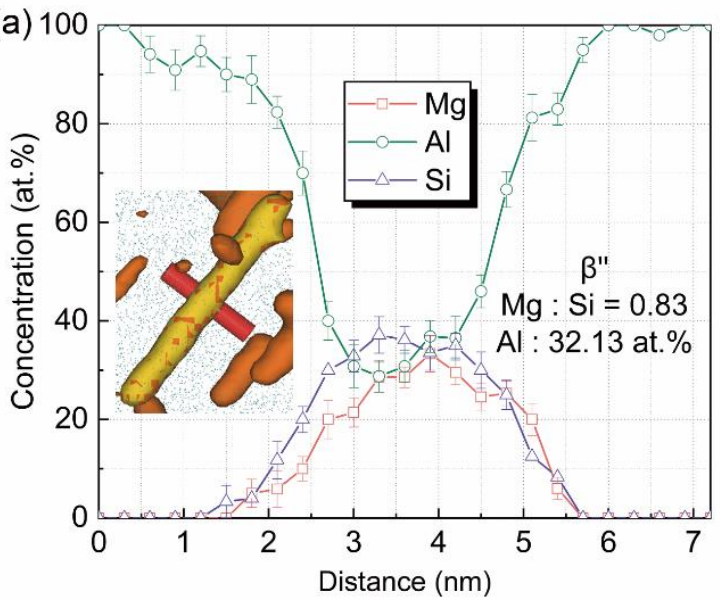

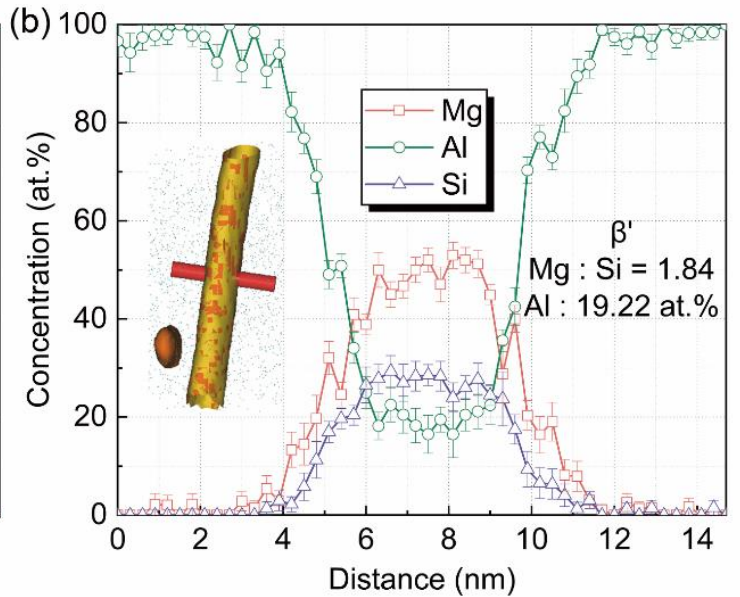

Fig.5 The representative 1D concentration distribution of (a) $\beta^{\prime \prime}$ and (b) $\beta^{\prime}$ marked in yellow in Fig.4 (a), (c), respectively. The 1D concentration profiles were measured by a selected cylinder with a moving step of $0.3 \mathrm{~nm}$. 

strength is conducted. The yield strength $\sigma_{y}$ of the Al-Mg-Si alloys consists of precipitation hardening $\sigma_{p}$, solid solution strengthening $\sigma_{s}$ and lattice resistance $\sigma_{i}$. The overall yield strength calculation can be achieved by adding the various contributions as equation (1) [17-20]. The $\sigma_{p}$ can be explained mainly from the modulus strengthening $\sigma_{\text {mod }}$, chemical strengthening $\sigma_{c h e m}$, coherency strengthening $\sigma_{c o h}$ and $\sigma_{G C}$ which is the contribution from GC, as shown in equation (2) [17, 20-22]. Detailed information of equations and parameters for calculation is provided by the supplementary information. Input the data of Table 1 and Table S1, the calculation results of the various contributions are shown in Table 2. At the SNA-AA state, the yield strength is mainly derived from contributions $56 \mathrm{MPa}$ to the yield strength while $0.21 \% \beta^{\prime \prime}$ contributes $119 \mathrm{MPa}$. At the LNA-AA state, $\beta^{\prime}$ with a volume fraction of $0.16 \%$ contributions $54 \mathrm{MPa}$ to the yield strength while $0.14 \% \beta^{\prime \prime}$ contributes $101 \mathrm{MPa}$. It fully demonstrates that $\beta^{\prime \prime}$ is the main strengthening phase in Al-Mg-Si alloy and the appearance of large-sized $\beta^{\prime}$ is a major cause of the negative NA effect. $\sigma_{y}^{\prime}$ is the yield strength obtained by the tensile test in Fig.1(b). $\sigma_{y}^{\prime}$ is similar to the calculation results which proves that the selection of the calculation formula is reasonable. Importantly, the relationship between strength and the size and volume fraction of nanoparticles is not linear which means that the comparison of strength increments per unit volume fraction at different states is not meaningful. 
183 Table 2. The calculation results of the various contributions

\begin{tabular}{|c|c|c|c|c|}
\hline \multicolumn{2}{|c|}{ Strength (MPa) } & SNA-AA & MNA-AA & LNA-AA \\
\hline \multirow{4}{*}{$\sigma_{\beta \prime \prime}$} & $\sigma_{m o d}$ & 14.6 & 7.8 & 6.5 \\
\hline & $\sigma_{\text {chem }}$ & 0.7 & 0.4 & 0.3 \\
\hline & $\sigma_{c o h}$ & 206.1 & 110.4 & 94.6 \\
\hline & SUM & 221 & 119 & 101 \\
\hline \multirow{4}{*}{$\sigma_{\beta}}$, & $\sigma_{m o d}$ & & 14.4 & 13.3 \\
\hline & $\sigma_{\text {chem }}$ & & 1.1 & 0.7 \\
\hline & $\sigma_{c o h}$ & & 40.5 & 40.1 \\
\hline & SUM & & 56 & 54 \\
\hline$\sigma_{G C}$ & & 2.3 & 3.6 & 4.2 \\
\hline$\sigma_{s}$ & & 44.0 & 49.1 & 53.8 \\
\hline$\sigma_{i}$ & & 10 & 10 & 10 \\
\hline$\sigma_{y}$ & & 277 & 238 & 223 \\
\hline$\sigma_{y}^{\prime}$ & & 280 & 253 & 230 \\
\hline
\end{tabular}
curves of SNA and LNA are shown in Fig.6. The exothermic peak at $50-100^{\circ} \mathrm{C}$ should be 186 the formation of the clusters II which can be the nucleation sites of $\beta^{\prime \prime}[23,24]$. The trough at $200-250^{\circ} \mathrm{C}$ can be related to the dissolution of unstable clusters I formed during NA [25]. The peak around $250^{\circ} \mathrm{C}$ and $300^{\circ} \mathrm{C}$ are normally associated with the formation of $\beta^{\prime \prime}$ and $\beta^{\prime}$, respectively $[23,25]$. LNA has no exothermic peak of clusters II. Combining the

190 APT results in Fig.3, it can be concluded that the LNA forms a lot of clusters I and 
consumes more solute atoms then does not have enough driving force to precipitate clusters II. The trough of LNA at $200-250^{\circ} \mathrm{C}$ caused by the dissolution of clusters I delayed the precipitation process of nanoparticles, leading to the peak of $\beta$ " move toward a higher temperature.

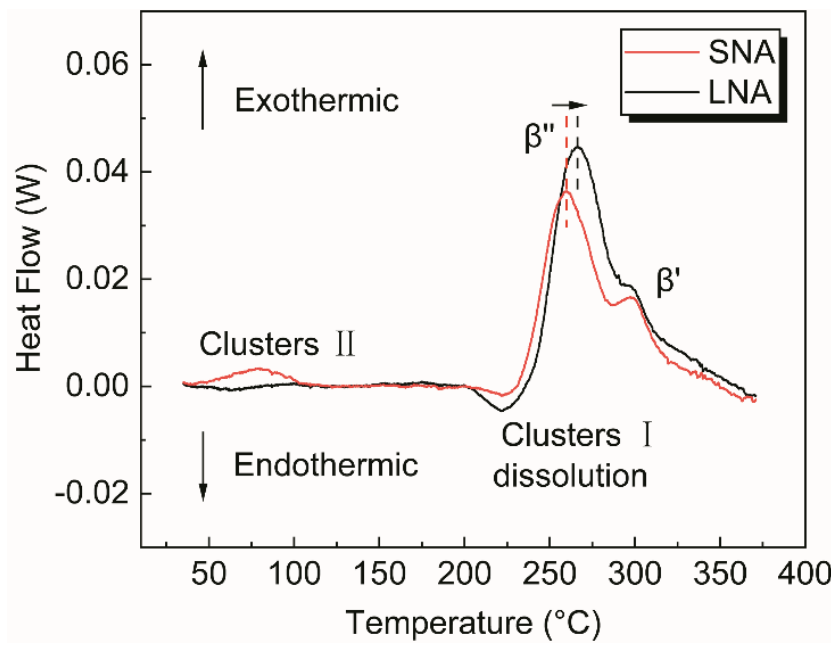

Fig.6 DSC flow curves of the Al-Mg-Si alloy at SNA and LNA.

Based on the above discussion, the AA process of SNA and LNA have been summarized through the schematic diagram in Fig.7. Solute atoms of SNA hardly aggregated and are evenly distributed as shown in Fig.3 (a), (c). During initial AA, sufficient solute atoms quickly precipitate a large amount of cluster II which acts as nucleation sites to form fine and dense $\beta^{\prime \prime}$ during the peaking AA. LNA formed numerous clusters I which will dissolve into the matrix during initial AA. Few clusters II formed during initial AA as shown in Fig.6. It takes time to dissolve cluster I and only when the solute supersaturation in the matrix reaches the nucleation condition, the formation of cluster II can occur. Under this condition, the distance between clusters II is usually very large. With the extending AA time, preferential growth of $\beta^{\prime \prime}$ on the few clusters II occur 
207 then converted to $\beta^{\prime}$. This preferential growth process is easier to capture the surrounding solute atoms, which directly restricts the growth of the GC. Additionally, the solute atoms in the region nearly which there is no nucleation site do not have enough driving force to

210 diffuse and precipitate but stay in the matrix. This is the fundamental reason for the

211 significant decrease in the volume fraction of the nanoparticles in LNA-AA.
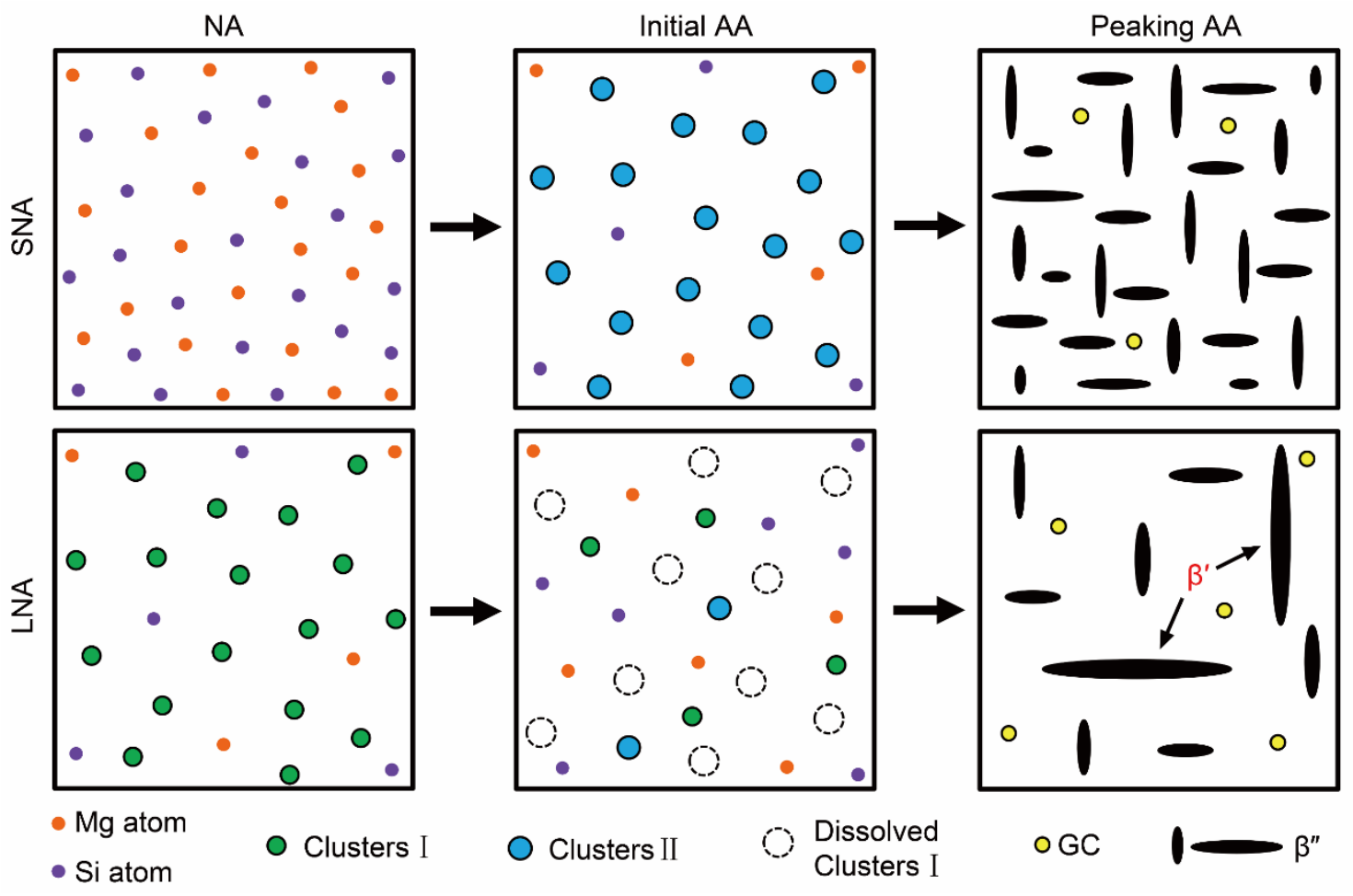

Fig.7 The schematic diagram of the AA process of SNA and LNA.

4. Conclusion

(1) We discovered that NA leads to the formation of different types of nanoparticles in AA Al-Mg-Si alloy meanwhile affect their fraction and dimension. The volume fraction of GC and $\beta^{\prime}$ is increasing with the extending of NA time, while the volume fraction of $\beta^{\prime \prime}$ is continuously declining from SNA-AA to LNA-AA. 
The $\beta^{\prime \prime}$ and $\beta^{\prime}$ become coarser in LNA-AA and the overall volume fraction drop from $0.74 \%$ to $0.37 \%$.

(2) Separation of the contribution from different types of nanoparticles to the alloy strength is conducted. At the SNA-AA state, the yield strength is mainly derived from $\sigma_{\beta^{\prime \prime}}$ which is $221 \mathrm{MPa}$. At the LNA-AA state, $\beta^{\prime}$ with a volume fraction of $0.16 \%$ contributions $54 \mathrm{MPa}$ to the yield strength while $0.14 \% \beta^{\prime \prime}$ contributes $101 \mathrm{MPa}$. It fully demonstrates that $\beta^{\prime \prime}$ is the main strengthening phase in Al-MgSi alloy and the appearance of large-sized $\beta^{\prime}$ is a major cause of the negative NA effect.

(3) LNA formed numerous clusters I which will dissolve into the matrix during initial AA. Few clusters II formed during initial AA. With the extending AA time, preferential growth of $\beta^{\prime \prime}$ on the few clusters II occurred then converted to $\beta^{\prime}$. This preferential growth process is easier to capture the surrounding solute atoms, which directly restricts the growth of the GC. Additionally, the solute atoms in the region nearly which there is no nucleation site do not have enough driving force to diffuse and precipitate but stay in the matrix. This is the fundamental reason for the significant decrease in the volume fraction of the nanoparticles in LNA-AA. 


\section{Abbreviations}

242 3D: Three-dimensional; APT: Atom probe tomography; HRTEM: High-resolution 243 transmission electron microscopy; TEM: Transmission electron microscopy; UA: Under 244 aging; DSC: differential scanning calorimeter.

\section{Declarations}

\section{Availability of data and materials}

248 All datasets are presented in the main paper or in additional supporting files.

\section{Competing interests}

250 The authors declare that they have no known competing financial interests or 251 personal relationships that could have appeared to influence the work reported in this 252 paper.

\section{Funding}

254 The National Key R\&D Program of China (No. 2016YFB1200602)

255 The Strategic Priority Program of the Chinese Academy of Sciences (No. XDB22020000)

256 Shenyang Key R\&D and technology transfer program (Z19-1-004).

\section{Authors' contributions}

258 Zhenjie Cui: Conceptualization, Methodology, Investigation, Formal analysis, Validation, 259 Writing - Original Draft.

260 Haichang Jiang: Conceptualization, Writing - review \& editing.

261 Duo Zhang: Methodology, Validation. 
262 Yuanyuan Song: Resources, Methodology.

263 Desheng Yan: Resources.

264 Lijian Rong: Supervision, Conceptualization, Writing - review \& editing.

265 Acknowledgment

266 The authors acknowledge the help from Duo Zhang and Qiyu Wang in the 267 experiments.

\section{Authors' information}

$269{ }^{1}$ CAS Key Laboratory of Nuclear Materials and Safety Assessment, Institute of Metal 270 Research, Chinese Academy of Science, Shenyang 110016, China

$271 \quad{ }^{2}$ School of Materials Science and Engineering, University of Science and Technology of 272 China, Shenyang 110016, China

$273{ }^{3}$ Shi-changxu Innovation Center for Advanced Materials, Institute of Metal Research, 274 Chinese Academy of Sciences, Shenyang 110016, China

275 Zhenjie Cui: Email address: zjcui17s@imr.ac.cn

276 Duo Zhang: Email address: dzhang15s@imr.ac.cn

277 Yuanyuan Song: Email address: $\underline{\text { songyuanyuan@imr.ac.cn }}$

278 Desheng Yan: Email address: dsyan@imr.ac.cn

$279 *$ Corresponding author:

280 Prof. Haichang Jiang, Tel: +86 0242397 1976; Email address: $\underline{\text { hcjiang@,imr.ac.cn }}$

281 Prof. Lijian Rong, Tel: +86 0242397 1979; Email address: 1jrong@imr.ac.cn 


\section{References}

284 [1] L. Ding, Z. Jia, J.-F. Nie, Y. Weng, L. Cao, H. Chen, X. Wu, Q. Liu, The structural 285 and compositional evolution of precipitates in Al-Mg-Si-Cu alloy, Acta Mater. 145 (2018) $286 \quad 437-450$.

[2] G.A. Edwards, K. Stiller, G.L. Dunlop, M.J. Couper, The precipitation sequence in Al-Mg-Si alloys, Acta Mater. 46(11) (1998) 3893-3904.

[3] S.J. Andersen, C.D. Marioara, J. Friis, S. Wenner, R. Holmestad, Precipitates in aluminium alloys, Adv. Phys-X 3(1) (2018).

[4] K. Buchanan, K. Colas, J. Ribis, A. Lopez, J. Garnier, Analysis of the metastable precipitates in peak-hardness aged $\mathrm{Al}-\mathrm{Mg}-\mathrm{Si}(-\mathrm{Cu})$ alloys with differing $\mathrm{Si}$ contents, Acta Mater. 132 (2017) 209-221.

[5] J. Røyset, T. Stene, J.A. Sæter, O. Reiso, The Effect of Intermediate Storage Temperature and Time on the Age Hardening Response of Al-Mg-Si Alloys, Mater. Sci. Forum 519-521 (2006) 239-244.

[6] G.H. Tao, C.H. Liu, J.H. Chen, Y.X. Lai, P.P. Ma, L.M. Liu, The influence of Mg/Si ratio on the negative natural aging effect in $\mathrm{Al}-\mathrm{Mg}-\mathrm{Si}-\mathrm{Cu}$ alloys, Mat. Sci. Eng A-Struct 642 (2015) 241-248.

[7] W. Tu, J. Tang, L. Ye, L. Cao, Y. Zeng, Q. Zhu, Y. Zhang, S. Liu, L. Ma, J. Lu, B. Yang, Effect of the natural aging time on the age-hardening response and precipitation behavior of the Al-0.4Mg-1.0Si-(Sn) alloy, Mater. Design (2020) 109307. 
precipitates in 12 orientations in Al-Mg-Si alloy, Scripta Mater. 62(9) (2010) 705-708.

[9] R. Vissers, M.A. van Huis, J. Jansen, H.W. Zandbergen, C.D. Marioara, S.J. Andersen,

The crystal structure of the $\beta^{\prime}$ phase in Al-Mg-Si alloys, Acta Mater. 55(11) (2007) 38153823.

[10] G. Sha, H. Möller, W.E. Stumpf, J.H. Xia, G. Govender, S.P. Ringer, Solute nanostructures and their strengthening effects in $\mathrm{Al}-7 \mathrm{Si}-0.6 \mathrm{Mg}$ alloy F357, Acta Mater. 60(2) (2012) 692-701.

[11] S. Zhu, H.-C. Shih, X. Cui, C.-Y. Yu, S.P. Ringer, Design of solute clustering during thermomechanical processing of AA6016 Al-Mg-Si alloy, Acta Mater. 203 (2021).

[12] P. Dumitraschkewitz, P.J. Uggowitzer, S.S.A. Gerstl, J.F. Löffler, S. Pogatscher, 314 Size-dependent diffusion controls natural aging in aluminium alloys, Nat. Commun. 10(1) 315 (2019). 139874. 
interrupted-aged Al-Mg-Si-Cu alloy, Philos. Mag. 88(3) (2008) 373-390.

[17] S. Esmaeili, D.J. Lloyd, W.J. Poole, Modeling of precipitation hardening for the naturally aged Al-Mg-Si-Cu alloy AA6111, Acta Mater. 51(12) (2003) 3467-3481.

[18] R.K.W. Marceau, A. de Vaucorbeil, G. Sha, S.P. Ringer, W.J. Poole, Analysis of strengthening in AA6111 during the early stages of aging: Atom probe tomography and yield stress modelling, Acta Mater. 61(19) (2013) 7285-7303.

[19] M.J. Starink, L.F. Cao, P.A. Rometsch, A model for the thermodynamics of and strengthening due to co-clusters in Al-Mg-Si-based alloys, Acta Mater. 60(10) (2012) 4194-4207.

[20] M. Yang, H. Chen, A. Orekhov, Q. Lu, X. Lan, K. Li, S. Zhang, M. Song, Y. Kong, D. Schryvers, Y. Du, Quantified contribution of $\beta^{\prime \prime}$ and $\beta^{\prime}$ precipitates to the strengthening of an aged Al-Mg-Si alloy, Mat. Sci. Eng A-Struct 774 (2020).

[21] A.J. ARDELL, Precipitation Hardening, Metall. Trans. A 16A (1985) 1985-2131.

[22] M. Song, Modeling the hardness and yield strength evolutions of aluminum alloy with rod/needle-shaped precipitates, Mat. Sci. Eng A-Struct 443(1-2) (2007) 172-177.

[23] C.S.T. Chang, J. Banhart, Low-Temperature Differential Scanning Calorimetry of an Al-Mg-Si Alloy, Metall. Mater. Trans. A 42(7) (2011) 1960-1964.

[24] V. Noseda Grau, A. Cuniberti, A. Tolley, V. Castro Riglos, M. Stipcich, Solute clustering behavior between $293 \mathrm{~K}$ and $373 \mathrm{~K}$ in a 6082 Aluminum alloy, J. Alloy Compd. 684 (2016) 481-487.

[25] A.K. Gupta, D.J. Lloyd, S.A. Court, Precipitation hardening in Al-Mg-Si alloys with 
346 and without excess Si, Mat. Sci. Eng A-Struct 316(1) (2001) 11-17. 


\section{Supplementary Files}

This is a list of supplementary files associated with this preprint. Click to download.

- SupplementaryInformation.docx 